\title{
-NOTES-
}

\section{ON TYPES OF CONVERGENCE AND ON THE BEHAVIOR OF APPROXIMATIONS IN THE NEIGHBORHOOD OF A MULTIPLE ROOT OF AN EQUATION*}

\author{
Br E. BODEWIG (The Hague)
}

1. Introduction. A chief problem of practical analysis consists in computing zeros of functions. For this, successive approximations are used. There are procedures in which the initial estimate is arbitrary and will always yield the nearby zero-point as the limit of a sequence of approximations. But there are only a few such procedures, the method of Laguerre being one. In almost all other cases the first estimate must satisfy some conditions in order that the sequence of approximate values will converge. How to obtain a useful first approximation is a special problem and has nothing to do with the method of approximation itself.

Now there are two gaps in the theory of all methods of approximations. First, it is only proved that the method will converge; there is no possibility of comparing different methods of solving the same problem. Until now, one was satisfied to demonstrate the procedures by one or several examples and, from these special examples, general conclusions about the method were drawn. Such conclusions are false, of course.

Second, it is supposed that the convergence of the same method will be similar in all circumstances. The problem was not even stated as to whether the convergence could depend on the multiplicity of the zero which is to be approximated. On the contrary, one assumed tacitly that this would not be the case, which is false again.

Both gaps occur for the same reason, namely that until now only the notion of convergence was introduced, with no measure of the convergence. With such a measure both problems can be solved in principle: several approximation methods can be compared, and it can be decided if the character of the convergence of the same method will always be the same.

2. A measure of the convergence of a sequence. A measure for the rapidity of the convergence of a sequence has first been given by the author. ${ }^{1}$

Definition. A convergent sequence

$$
x_{1}, x_{2}, x_{3}, \cdots, x_{n}, \cdots \rightarrow X
$$

with the limit $X$ is called "convergent in the degree $g$ " if

$$
\lim _{n \rightarrow \infty} \frac{x_{n+1}-X}{\left(x_{n}-X\right)^{\circ}}=c \neq 0 \text {. }
$$

Introducing the deviations of the terms from their limit, $x_{n}-X=d_{n}$, this becomes

$$
\lim \frac{d_{n+1}}{d_{n}^{0}}=c \neq 0 .
$$

To determine the degree of convergence we have, therefore, to develop $d_{n+1}$ in

*Received June 21, 1948.

'E. Bodewig, Sur la méthode de Laguerre, Akad. Wetensch. Proc. (Amst.) 49, 911-21, especially 912 (1946). 
powers of $d_{n}$. The lowest term $c d_{n}^{o}$ of this development determines the degree $g$ and the coefficient $c$.

The larger $g$ is, the more rapidly the sequence will converge. Briefly we can say that, if $k$ is the number of exact decimals of $x_{n}$, then the number of exact decimals of $x_{n+1}$ will be $\mathrm{kg}$. Whatever the coefficient $c$ may be, therefore, a method of approximation will be more effective, in the long run, than any other method with a lower degree of convergence. Only in the beginning of the sequence may the influence of the coefficient $c$ change this behavior.

For the reasons given in the introduction, the author believes the notion of degree of convergence to be of fundamental importance in numerical analysis.

The reciprocal of a linear operator. We give first a general example and seek a process which will yield the reciprocal of a linear operator by a sequence of successive approximations converging in degree $k$.

For this purpose we consider first such a process for the reciprocal of a number $N$. Here $X=1 / N$, and by our definition we must have

$$
d_{n+1}=c d_{n}^{k}+\cdots
$$

or

$$
x_{n+1}-1 / N=c\left(x_{n}-1 / N\right)^{k}+\cdots .
$$

The constant $c$ is still arbitrary. It has to be chosen in such a manner that all powers of the unknown $1 / N$ will be cancelled. This is done by letting $c=N^{k-1}$. Thus we have

$$
x_{n+1}=x_{n}\left[k-\left(\begin{array}{l}
k \\
2
\end{array}\right) z_{n}+\left(\begin{array}{l}
k \\
3
\end{array}\right) z_{n}^{2}-\cdots \pm z_{n}^{k-1}\right], \quad \text { where } \quad z_{n}=N x_{n} \text {. }
$$

The sequence $x_{1}, x_{2}, \cdots$ will converge if

$$
0<x_{1}<2 / N
$$

and the convergence will be of degree $k$.

For $k=2$ the known formula

$$
x_{n+1}=x_{n}\left(2-N x_{n}\right)
$$

results, which converges quadratically.

The same formula will also hold for any linear operator, since the proof did not use any properties other than those which are true for every linear operator. But the conditions of convergence must be established for every operator separately.

So the formulas hold if $N$ is a matrix. This case has been investigated by G. Schulz ${ }^{2}$ and again by other authors independently. In a former paper ${ }^{3}$ the author has given another proof of the formulas slightly different from the above proof.

3. Newton's formula and generalizations. First we give a generalization of Newton's formula. While the usual Newton formula is customarily obtained by means of Taylor's theorem, we shall proceed here in another way.

${ }^{2} \mathrm{G}$. Schulz, Iterative Berechnung einer reziproken Matrix, Zeits. angew Math. Mech. 13, 57-59 (1933).

${ }^{3} \mathrm{E}$. Bodewig, Bericht über die verschiedenen Methoden zur Lösung eines Systems linearer Gleichungen mit reellen Koeffizienten, Akad. Wetensch. Proc. (Amst.) 50, 930-941, 1104-1116, 1285-1295 (1947) and 51, 53-64, 211-219 (1948). 
Let $f(x)$ have the root $X$ of multiplicity $p$ :

$$
f(x)=(x-X)^{p} \cdot g(x), \quad \text { where } \quad g(X) \neq 0 .
$$

Then

$$
f^{\prime}(x)=p(x-X)^{p-1} g+(x-X)^{p} g^{\prime}
$$

or

$$
\frac{f^{\prime}(x)}{f(x)}=\frac{p}{x-X}+\frac{g^{\prime}(x)}{g(x)}
$$

Near $x=X$ the first term on the right is very large, while the second one is "finite". So with very good approximation,

$$
\frac{f^{\prime}(x)}{f(x)}=\frac{p}{x-X}
$$

that is

$$
X \approx x-p \frac{f(x)}{f^{\prime}(x)} .
$$

Thus the general Newton's formula in the neighborhood of a root of multiplicity $p$ is

$$
x_{n+1}=x_{n}-p \frac{f\left(x_{n}\right)}{f^{\prime}\left(x_{n}\right)} .
$$

More generally, we set

$$
x_{n+1}=x_{n}-a \frac{f\left(x_{n}\right)}{f^{\prime}\left(x_{n}\right)}
$$

and determine the character of the convergence of the sequence

$$
x_{1}, x_{2}, x_{3}, \cdots, x_{n}, \cdots \rightarrow X
$$

which we suppose converges, the conditions on $x_{1}$ being unknown. Developing $g(x)$ at $x=X$ we obtain from (1) that

$$
\begin{aligned}
f\left(x_{n}\right) & =\left(x_{n}-X\right)^{p} g(X)+\left(x_{n}-X\right)^{p+1} g^{\prime}(X)+\cdots \\
& =d_{n}^{p} g(X)+d_{n}^{p+1} g^{\prime}(X)+\cdots
\end{aligned}
$$

or by $\left(3^{\prime}\right)$,

$$
x_{n+1}=x_{n}-\frac{a}{p} d_{n}+\frac{a}{p^{2}} \frac{g^{\prime}(X)}{g(X)} d_{n}^{2}+\cdots .
$$

Subtracting $X$ from both sides, we get

$$
d_{n+1}=\left(1-\frac{a}{p}\right) d_{n}+\frac{a}{p^{2}} \frac{g^{\prime}(X)}{g(X)} d_{n}^{2}+\cdots .
$$

Here the linear term of $d_{n}$ will be cancelled only if $a=p$. So we have the following results. 
Result 1 . In the neighborhood of a root of multiplicity $p$ formula $\left(3^{\prime}\right)$ will yield a sequence which converges quadratically to the root (if it converges at all) only for $a=p$. The convergence is better the larger $p$ is.

2. For $a \neq p$, however, the sequence is only linearly convergent; the smaller the quotient $a / p$ is, the worse the convergence. So Newton's formula will not give better convergence in this case than, for instance, the rule of false position, which is often simpler.

3. The usual Newton formula (having $a=1$ ) will therefore converge quadratically only near a simple root, but linearly near a multiple root.

4. If it is known that the root is multiple (for instance by observing that $f^{\prime}\left(x_{1}\right)$ is small) but the exact multiplicity is not known, we put $a=2$ or perhaps $a=3$.

A formula which always yields quadratic convergence, without requiring knowledge of the multiplicity of the roots, is obtained by choosing a function which has all the roots of $f(x)$, with each of them simple. This is $f / f^{\prime}$. Then $\left(3^{\prime}\right)$ with $a=1$ will yield

$$
x_{n+1}=x_{n}-\frac{f f^{\prime}}{f^{\prime 2}-f f^{\prime \prime}},
$$

where on the right side the argument $x_{n}$ has been omitted. The calculation is simpler if we put (4) in the form

$$
x_{n+1}=x_{n}-\frac{f}{f^{\prime}-A} \quad \text { with } \quad A=f f^{\prime \prime} / f^{\prime} .
$$

More generally we put

$$
x_{n+1}=x_{n}-\frac{f f^{\prime}}{f^{\prime 2}-a f f^{\prime \prime}}
$$

and determine the character of the convergence for the case that

$$
f(x)=(x-X)^{p} g(x) .
$$

Proceeding as above, we obtain

$$
d_{n+1}=c_{1} d_{n}+c_{2} d_{n}^{2}+\cdots,
$$

where

$$
c_{1}=-(p-1)(a-1) /(p+a-a p), \quad c_{2}=-(a+a p-p) g^{\prime}(X) / p(p+a-a p)^{2} g(X) .
$$

So $c_{1}$ vanishes in the cases $a=1$ and $p=1$. The first case yields formula (4). In the second case also $c_{2}$ will vanish if $a=1 / 2$. So we have the results below.

Result 1. Formula (4) yields a convergence which is always quadratic. Since the factor $c_{2}$ is equal to $g^{\prime}(X) / p g(X)$, the convergence is least good in the case of a simple root; the greater the multiplicity $p$ of the root, however, the better the convergence.

2. The quadratic convergence of (4) for a root of multiplicity $p$ is worse than that of (3) if $p \neq 1$ and is the same if $p=1$.

3. The simple formula (3) is therefore always preferable to (4), though only applicable if $p$ is known.

4. In the case of a simple root, formula (5), with $a$ arbitrary, will always yield quadratic convergence. It is, therefore, no better than the usual Newton formula. The Newton formula, moreover, is preferable as it does not contain $f^{\prime \prime}$. 
5. Formula (5) with $a=1 / 2$ yields cubic convergence in the case of a simple root, with

$$
c_{3}=-\frac{1}{2} \frac{g^{\prime \prime}(X)}{g(X)}+\left[\frac{g^{\prime}(X)}{g(X)}\right]^{2},
$$

but only linear convergence with $c_{1}=(p-1) /(p+1)$ in the case of a multiple root. Hence it is no better a formula than the simple Newton formula would be.

6. A convergence which is always cubical, is obtained by taking the average of the results of formulas (3) and (4). For the coefficients $c_{2}$ are absolutely equal, but of opposite sign in both formulae.

The simplified Newton method. It is known that the usual Newton formula $x_{n+1}=$ $x_{n}-f\left(x_{n}\right) / f^{\prime}\left(x_{n}\right)$ is often simplified to

$$
x_{n+1}=x_{n}-\frac{f\left(x_{n}\right)}{f^{\prime}\left(x_{1}\right)}
$$

with constant denominator. Some authors (for instance Whittaker-Robinson) consider formula (6) to be nearly as effective as the usual one. This is, however, not the case. For it is easily seen that every sequence

$$
x_{n+1}=x_{n}-c f\left(x_{n}\right)
$$

yields $d_{n+1}=d_{n}-c f\left(x_{n}\right)=d_{n}-c d_{n}^{p} g(X)+\cdots$, and so always converges linearly for every $p$.

The same result has been given by Ostrowski. ${ }^{4}$ The present proof is much simpler. It must be added, however, that for Ostrowski the chief point is to give the conditions for convergence.

Example. To illustrate the difference between quadratic and cubic convergence we take an example of H. S. Wall. ${ }^{5}$ The square root of $b$ is calculated by means of Newton's formula and by means of (5). Taking $f(x)=x^{2}-b$ we obtain the two formulas:

$$
\begin{array}{lll}
x_{n+1}=\frac{1}{2}\left(x_{n}+b / x_{n}\right) & \text { with } & c_{2}=1 / 2 b^{1 / 2}, \\
x_{n+1}=\left(x_{n}^{2}+3 b\right) x_{n} /\left(3 x_{n}^{2}+b\right) & \text { with } & c_{3}=1 / 4 b .
\end{array}
$$

Putting $b=2$ and $x_{1}=1$ we obtain the sequences where all figures are correct and only the last ones are slightly different:

\begin{tabular}{lll} 
& \multicolumn{1}{c}{$\begin{array}{c}\text { quadratic } \\
\text { convergence }\end{array}$} & \multicolumn{1}{c}{$\begin{array}{c}\text { cubic } \\
\text { convergence }\end{array}$} \\
$x_{1}$ & 1 & 1 \\
$x_{2}$ & 1.5 & 1.40 \\
$x_{3}$ & 1.417 & 1.4142132 \\
$x_{4}$ & 1.414216 & 1.414213562373095048796 \\
$x_{5}$ & 1.414213562375 &
\end{tabular}

${ }^{4}$ A. Ostrowski, Über eine Modifikation des Newtonschen Näherungsverfahrens, Akad. Nauk. SSSR (Gruz, Fil Trudy) 2, 241-249 (1937).

'H. S. Wall, A modification of Newton's method, Amer. Math. Monthly 55, 90-91 (1948). 
Starting from $x_{1}=10$ we have the sequences:

$\begin{array}{lcc} & \begin{array}{c}\text { quadratic } \\ \text { convergence }\end{array} & \begin{array}{c}\text { cubic } \\ \text { convergence }\end{array} \\ x_{1} & 10 & 10 \\ x_{2} & 5 & 3.5 \\ x_{3} & 2.7 & 1.6 \\ x_{4} & 1.7 & 1.415 \\ x_{5} & 1.44 & 1.414213564\end{array}$

4. Laguerre's method for algebraic equations and related formulas. While Newton's method and the generalized methods above are applicable to all equations and hold for real and complex roots, Laguerre ${ }^{6}$ has given an elegant method which applies only to algebraic equations having all real roots. The sequence is defined by

$$
x_{k+1}=x_{k}-\frac{n f\left(x_{k}\right)}{f^{\prime}\left(x_{k}\right) \pm\left[H\left(x_{k}\right)\right]^{1 / 2}}
$$

where $n$ is the degree of the equation $f(x)=0$ and $H(x) \equiv(n-1)^{2} f^{2}-n(n-1) f f^{\prime \prime}=$ Hesse's covariant of $f$. The sign of the square root can be taken as either + or - , for the method approximates two roots simultaneously.

The method has been analyzed in an earlier paper of the author. ${ }^{7}$ The features of the method are:

1. Laguerre proved that the method always converges, i.e. $x_{1}$ is arbitrary (in contrast to Newton's method, for example, wherein $x_{1}$ must satisfy certain conditions).

2. Laguerre proved that each sign of the square root yields a convergent sequence:

$$
\begin{aligned}
& x_{1}, x_{2}^{\prime}, x_{3}^{\prime}, \cdots, x_{k}^{\prime}, \cdots \rightarrow X^{\prime} \\
& x_{1}, x_{2}^{\prime \prime}, x_{3}^{\prime \prime}, \cdots, x_{k}^{\prime \prime}, \cdots \rightarrow X^{\prime \prime}
\end{aligned}
$$

where $X^{\prime}$ is the nearest root lying to the left of $x_{1}$ and $X^{\prime \prime}$ the nearest root lying to the right of $x_{1}$. This will always hold if the straight line is considered closed at infinity.

3. The author has proved that the convergence is cubic in the case of a simple root, but only linear in the case of a multiple root.

4. In order to obtain cubic convergence in the case of a root of multiplicity $p$, the author proved that in the above formula, $H\left(x_{k}\right)$ is to be replaced by $a H\left(x_{k}\right)$ where $a=(n-p) / p(n-1)$.

5. Later van der Corput ${ }^{8}$ proved that if the equation has at least one root of multiplicity $\geqq p$, the formula mentioned which replaces $H(x)$ by $a H(x)$ will approximate only the two roots of multiplicity $\geqq p$ lying to the left and to the right of $x_{1}$, thus skipping the roots of multiplicity $<p$. In the case of a root of multiplicity $p$ the convergence is cubic, whereas in the case of a root of multiplicity $>p$ it is only linear.

5. The general convergence of degree $n$. In the preceding some important examples

${ }^{6} \mathrm{E}$. N. Laguerre, Sur une méthode pour obtenir par approximation les racines d'une équation algébrique qui a toutes ses racines réelles, Oeuvres de Laguerre 1, 87-103 (1898).

${ }^{7}$ See footnote 1.

${ }^{8}$ J. G. van der Corput, Sur l'approximation de Laguerre des racines d'une équation algébrique qui a toutes ses racines réelles, Akad. Wetensch. Proc. (Amst.) 49, 922-929 (1946). 
have been given showing the difference in convergence near a simple and a multiple root. This will now be generalized.

For this purpose we suppose we have a process $F(x)$ yielding to every value $x_{k}$ a better value $x_{k+1}$ :

$$
x_{k+1}=F\left(x_{k}\right) \text {. }
$$

We are not concerned here with the conditions for convergence itself of the sequence, but merely with the character of the convergence. Now we seek the general function $F(x)$ which yields a sequence converging in degree $n$. Then

$$
x_{k+1}-X=c\left(x_{k}-X\right)^{n}+\cdots \text {. }
$$

Now when $D^{i} \equiv d^{i} / d x^{i}$ is the $i$ th derivate,

$$
D^{i} F\left(x_{k}\right)=D^{i} x_{k+1}=c n(n-1) \cdots(n-i+1)\left(x_{k}-X\right)^{n-i}+\cdots .
$$

Putting $x_{k}=X$, the right side will vanish if $i<n$. So

$$
D^{i} F(X)=0 \quad \text { for } \quad i=1,2, \cdots, n-1 .
$$

This is the modified condition that the sequence $x_{1}, x_{2}, \cdots$ converges in degree $n$.

Before determining $F(x)$ we conclude from (8) that

$$
X=\lim x_{k+1}=\lim F\left(x_{k}\right)=F\left(\lim x_{k}\right)=F(X),
$$

that is

$$
X=F(X) \text {. }
$$

This condition holds for every $\operatorname{root} X$ of $f(x)=0$. Therefore

$$
F(x)=x+f(x) g(x),
$$

where $g(x)$ is arbitrary.

Now if (9) is to hold for $i=1$, the sequence $x_{k}$ must converge quadratically. Therefore, since

$$
\begin{gathered}
0=D F(X)=1+f^{\prime}(X) g(X), \\
g(X)=-1 / f^{\prime}(X) \quad \text { or } \quad g(x)=-1 / f^{\prime}(x)+f(x) g_{1}(x)
\end{gathered}
$$

and the function (11) becomes

$$
F(x)=x-f(x) / f^{\prime}(x)+f^{2}(x) g_{1}(x) .
$$

It will yield a quadratically convergent sequence for an arbitrary $g_{1}(x)$.

Now if (9) has to hold for $i=2$, that is if the sequence has to converge cubically, we obtain

$$
0=D^{2} F(X)=-D^{2}\left(f / f^{\prime}\right)+D^{2}\left(f^{2} g_{1}\right) .
$$

Here $f^{2} g_{1}$ is differentiated by Leibnitz' rule, and in the result $x$ is replaced by $X$. Then all terms will vanish, except the last one: $g_{1} D^{2}\left(f^{2}\right)=g_{1}(X) \cdot 2 f^{\prime 2}(X)$. On the other hand since $f(X)=0$, we have for $x=X$, if for brevity we set $r=1 / f^{\prime}$,

$$
D^{2}\left(f / f^{\prime}\right)=D^{2}(f r)=2 D f \cdot D r+r D^{2} f=-2 f^{\prime} f^{\prime \prime} r^{2}+f^{\prime \prime} r=-f^{\prime \prime} r .
$$


Therefore

$$
0=D^{2} F(X)=f^{\prime \prime}(X) \cdot r(X)+2 f^{\prime 2}(X) g_{1}(X),
$$

or

$$
g_{1}(x)=-1 / 2 f^{\prime \prime}(x) r^{3}(x)+f(x) g_{2}(x)
$$

and

$$
F(x)=x-f r-1 / 2 f^{2} f^{\prime \prime} r^{3}+f^{3} g_{2},
$$

where $g_{2}(x)$ is arbitrary.

To obtain generally a sequence converging in degree $n$ we generalize (12) and (13) by means of the operator

$$
P=r \cdot D, \quad \text { where } \quad r=1 / f^{\prime}(x) \quad \text { and } \quad D=d / d x
$$

and write

$$
F(x)=F_{n}(x)-f^{n}(x) g_{n}(x),
$$

where

$$
F_{n}(x)=x-f r+\frac{1}{2} f^{2} \cdot \operatorname{Pr}-\frac{1}{6} f^{3} \cdot P^{2} r+\cdots+\frac{(-1)^{n-1}}{(n-1) !} f^{n-1} \cdot P^{n-2} r .
$$

We have to prove that this is the expression we obtain if we proceed from (13) to sequences which converge in degree $4,5, \cdots, n$. Since the form of $F_{2}$ and $F_{3}$ is identical with (12) and (13), respectively, we proceed by induction and assume that $F_{n}(x)$ is the true expression; we have to prove that then $F_{n+1}(x)$ is the expression yielding a sequence which converges in degree $n+1$.

Now since

$$
\begin{aligned}
D(f r)= & \text { Df }+f D r=1+f f^{\prime} \cdot \operatorname{Pr}, \\
D F_{n}(x)= & 1-\left(1+f f^{\prime} \cdot \operatorname{Pr}\right)+\left(f f^{\prime} \cdot \operatorname{Pr}+\frac{1}{2} f^{2} f^{\prime} \cdot P^{2} r\right)-\frac{1}{6}\left(3 f^{2} f^{\prime} \cdot P^{2} r\right. \\
& \left.\quad+f^{3} f^{\prime} \cdot P^{3} r\right)+\cdots+\frac{(-1)^{n-1}}{(n-1) !}\left[(n-1) ! f^{n-2} f^{\prime} \cdot P^{n-2} r+f^{n-1} f^{\prime} \cdot P^{n-1} r\right] \\
= & \frac{(-1)^{n-1}}{(n-1) !} f^{n-1} f^{\prime} \cdot P^{n-1} r .
\end{aligned}
$$

Then since

$$
D^{m}[f(X)]^{m}=m !\left[f^{\prime}(X)\right]^{m},
$$

it"follows that

$$
D^{n} F_{n}(X)=D^{n-1} D F_{n}(X)=(-1)^{n-1}\left[f^{\prime}(X)\right]^{n-1} f^{\prime}(X) \cdot P^{n-1} r .
$$

Furthermore,

$$
D^{n}\left\{[f(X)]^{n} g_{n}(X)\right\}=n !\left[f^{\prime}(X)\right]^{n} g_{n}(X),
$$


that is

$$
g_{n}(X)=\frac{1}{n !} P^{n-1} r \quad \text { q.e.d. }
$$

Although (14) and (14a) have been constructed to yield a sequence converging in degree $n$ (if it converges at all), we have to investigate what happens when the root $X$ is multiple. In this case the equation $0=D F(X)$ standing between Eqs. (11) and (12) can no longer be satisfied by any function $g(x)$ whatever. For we must have

$$
0=D F(X)=1+f^{\prime}(X) g(X)=1,
$$

since $f^{\prime}(X)=0$ and $X$ is multiple. So not even a quadratic convergence can be obtained, that is, the convergence is linear.

The formula (14) can be given another form which originally dates from Euler. ${ }^{9}$ He gave the following generalization of the method of Newton about which the author has reported in a former paper. ${ }^{10}$

For the function $y=f(x)$ a value $X$ has to be determined such that $y=0$. Using the inverse function $x=u(y), X$ is given by $X=u(0)$. But $u$ is unknown, and of $X$ only an approximate value $a$ is known. Thus, $f(a)$ is known. Now, for $A=f(a)$ Euler develops $u(0)$ in a Taylor series near $A$ :

$$
X=u(A-A)=u(A)-A u^{\prime}(A)+1 / 2 A^{2} u^{\prime \prime}(A)-1 / 6 A^{3} u^{\prime \prime \prime}(A)+\cdots .
$$

Here the derivates of $u$ for the argument $A$ are known, for

$$
\begin{gathered}
u(A)=a, \quad u^{\prime}(A)=d x / d y=1 / f^{\prime}(a)=r(a), \\
u^{\prime \prime}(A)=-f^{\prime \prime}(a) r^{3}(a), \quad u^{\prime \prime \prime}(A)=\left[3 f^{\prime \prime 2}(a)-f^{\prime}(a) f^{\prime \prime \prime}(a)\right] r^{5}(a), \cdots .
\end{gathered}
$$

Setting

$$
u^{(n)}(y)=(-1)^{n-1} U_{n}(x) r^{2 n-1}(x),
$$

we have the recurrence formula for $U_{n}$ :

$$
U_{n+1}=(2 n-1) f^{\prime \prime} U_{n}-f^{\prime} U_{n}^{\prime} .
$$

Now the first terms of (15) are identical with the corresponding terms of (14a). This must be proved true for all the terms. Thus we proceed again by induction and suppose that (15) holds for $n$ and prove that it will hold for $n+1$. Let therefore

$$
\begin{gathered}
P^{n} r=u^{(n)}(y), \quad \text { then } \\
P^{n+1} r=P P^{n} r=P u^{(n)}=r \cdot D u^{(n)}=(-1)^{n-1} r\left[U_{n}^{\prime} r^{2 n-1}-(2 n-1) f^{\prime \prime} U_{n} r^{2 n}\right] \\
=(-1)^{n} U_{n+1} r^{2 n+1}=u^{(n+1)}(y), \quad \text { q.e.d. }
\end{gathered}
$$

So we have the results following.

Result 1. The developments (14) and (15) are identical.

2. The convergence of the sequence $x_{1}, x_{2}, \cdots$ yielded by the function $F(x)$ of (14) and (14a) is of degree $n$ if the root $X$ to which it converges is simple, but only linear if the root is multiple.

${ }^{9} \mathrm{~L}$. Euler, Institutiones Calculi Differentialis, II, Cap. IX.-Opera Omnia, ser. I, vol. X, p. 422-455.

${ }^{10} \mathrm{E}$. Bodewig, Über das Eulersche Verfahren zur Aufösung numerischer Gleichungen, Commentarii Math. Helvetici 8, 1-4 (1935). 